\title{
Internal Jugular Vein Septic Thrombophlebitis (Lemierre Syndrome) as a Complication of Pharyngitis
}

\author{
Andrew P. Wong, MD, Maurice L. Duggins, MD, and Tara Neil, MD
}

Sore throat is a common presenting complaint in the outpatient setting. Most cases are nonbacterial in origin, but those that are bacterial are usually the result of group A $\boldsymbol{\beta}$-hemolytic streptococcus. Guidelines exist to help physicians decide whether to treat with an antibiotic. Lemierre syndrome is a dangerous potential sequela of pharyngitis that results in septic thrombophlebitis of the internal jugular (IJ) vein. A high index of suspicion is needed to consider this diagnosis in the workup of pharyngitis and should be aggressively treated once identified. Consideration should be given to completing blood cultures and neck imaging because of clinical suspicion. The case study discussed here illustrates the presentation, evaluation, and treatment of Lemierre syndrome. (J Am Board Fam Med 2015;28:425-430.)

Keywords: Fusobacterium, Fusobacterium nucleatum, Jugular Vein Thrombus, Lemierre Syndrome

\section{Case Description}

History of Present Illness

Anotherwise healthy 21-year-old African American women with a medical history of recurrent tonsillitis and Streptococcus pharyngitis presented to the emergency department (ED) with 7 days of sore throat, nausea, vomiting, and fever up to $102^{\circ} \mathrm{F}$. She had presented to the ED 3 days earlier for the same complaint, was diagnosed with pharyngitis, and was discharged from the hospital with analgesics and a 5-day course of azithromycin, without any interval improvement. She felt her current symptoms were similar to her previous episodes of pharyngitis but more severe. The review of systems was positive for fever, rigors, sweats, headaches, dizziness, weakness, swollen neck glands, right ear pain, decreased fluid intake, nausea, vomiting, loss

This article was externally peer reviewed.

Submitted 24 April 2014; revised 11 December 2014; accepted 18 December 2014.

From the Via Christi Family Medicine Residency, Wichita Center for Graduate Medical Education (APW) and the Via Christi Family Medicine Program (MLD, TN), Kansas University School of Medicine, Wichita.

Funding: none.

Conflict of interest: none declared.

Corresponding author: Maurice L. Duggins, MD, Via Christi Family Medicine Program, Kansas University School of Medicine, 1121 S. Clifton Ave. Wichita, KS 67218-2912 (E-mail: Maurice.Duggins@via-christi.org). of appetite, dysphagia secondary to pain, dry cough, palpitations, back pain, and orange urine discoloration. The remainder of her review of systems was negative.

\section{Medical, Surgical, Social, and Family Histories}

This patient's medical history was negative for any chronic diseases. She did have a history of $>10$ episodes of pharyngitis and tonsillitis over her lifetime. Surgical history was noncontributory. Social history was negative for tobacco, alcohol, and illicit drug use. She reported that her last menstrual period was 35 days before presentation to the $\mathrm{ED}$, and she denied any previous sexual activity, including intercourse or oral sex. As a college student, she was living in an apartment with a roommate and working part time as a cashier. Her family history was positive for type 2 diabetes mellitus.

\section{Medications and Allergies}

At the time of admission, the patient was taking hydrocodone with acetaminophen and azithromycin. Her allergies included an urticarial rash to penicillin.

\section{Physical Examination}

She was febrile, hypotensive, and tachycardic with a temperature of $102.5^{\circ} \mathrm{F}$, blood pressure of 99/61 $\mathrm{mmHg}$, and a pulse of 140 beats per minute. Res- 
pirations were 18 breaths per minute, with an oxygen saturation of $100 \%$ in room air. The patient reported 10 of 10 pain in her throat. Upon examination, she appeared ill and in moderate distress, but she was alert and had no neurological deficits or confusion. Pharyngeal examination revealed erythema with grade 3 tonsil hypertrophy without exudates and dry mucous membranes. Neck was tender upon palpation, with bilateral anterior cervical lymphadenopathy (right greater than left). Lungs were clear upon bilateral auscultation, but there were slightly diminished breath sounds diffusely. Heart auscultation revealed a tachycardic, regular rhythm without any murmur. Abdominal examination revealed a soft, nondistended abdomen that had mild diffuse tenderness with normal bowel sounds. A radial pulse deficit was noted, along with normal capillary refill, no peripheral edema, and no skin rashes.

The overall clinical presentation was a patient showing signs of sepsis following failed outpatient treatment of pharyngitis and tonsillitis. Despite aggressive fluid resuscitation with $3 \mathrm{~L}$ of normal saline, the patient remained hypotensive and tachycardic, with a blood pressure and pulse in the range of $80-90 / 50-70 \mathrm{mmHg}$ and $100-120$ beats per minute, respectively. Fever, pain, nausea, and vomiting were treated symptomatically.

The initial laboratory workup in the ED included a complete metabolic panel, complete blood count, lipase, urine pregnancy test, rapid group A streptococcus pharyngeal swab, heterophile anti- body screen for mononucleosis, urinalysis, microscopic urine examination, chest radiograph, and 2 blood cultures. Results were consistent with hypovolemia and a stress response. She had leukocytosis with a left shift, mild hyperglycemia, mild thrombocytopenia, and mild hyperbilirubinemia. Lipase, group A streptococcus swab, heterophile antibody screen, urine pregnancy test, and chest radiograph were all negative.

\section{Hospital Course}

The patient was admitted to the hospital and started on empiric antibiotic treatment with intravenous (IV) ceftriaxone ( $1 \mathrm{~g}$ every 24 hours). A respiratory virus panel was added to the initial lab work. Aggressive fluid hydration was continued as the patient continued to demonstrate a septic clinical picture.

On the second hospital day, antibiotic coverage was broadened in response to an increasing white blood cell count, as shown in Figure 1, which highlights pertinent lab trends and points of key medication changes during the hospital course. Clindamycin (600 mg IV every 8 hours) was added for anaerobic coverage (shown as the orange dashed line in Figure 1). Over the next 2 days, test results gradually showed improvement of white blood count, neutrophil count, and C-reactive protein concentration. The patient began to tolerate oral intake and was switched to oral cefdinir (300 mg twice daily) and clindamycin (300 mg every 8 hours).

Despite the improvement in lab values, she began to develop worsening right-sided neck pain

Figure 1. Trends in pertinent laboratory values during the hospital course. The patient was discharged from the hospital on day 7. Laboratory values on day 12 are after discharge. The dashed orange line marks the initiation of clindamycin. The dotted-dashed orange line marks the change in antibiotics to high-dose ceftriaxone, vancomycin, and metronidazole. The dotted black line marks the initiation of dexamethasone taper. ANC, absolute neutrophil count; ESR, erythrocyte sedimentation rate; CRP, C-reactive protein; WBC, white blood cells.

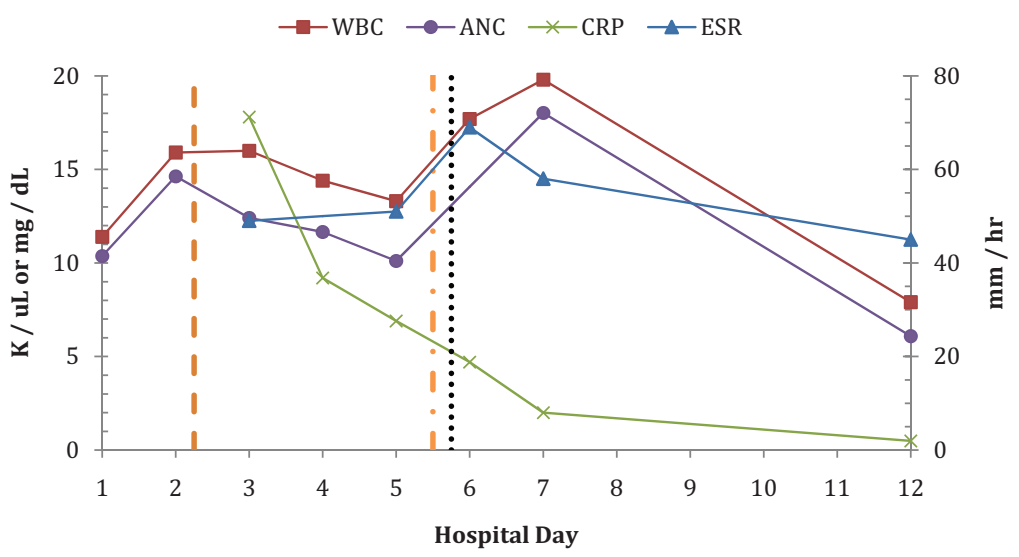


with increased swelling, warmth, induration, and mild trismus. Strep cultures were negative and the respiratory virus panel was positive for a concurrent rhinovirus infection. On the fourth hospital day, 1 of 2 blood cultures were positive for gram-negative rods. Her right neck swelling continued to worsen, and on the fifth hospital day, computed tomography (CT) of the neck and soft tissues with and without IV contrast was ordered to evaluate for a possible abscess. CT revealed complete occlusion of the right IJ vein, extending from just inferior to the IJ foramen to the lower neck, with associated retropharyngeal edema (Figure 2). There was a question of whether the hypodense area in the retropharyngeal space represented edema or an abscess (Figure 2C).

On the fifth hospital day, the initial blood cultures identified an anaerobic organism, Fusobacterium nucleatum, making the diagnosis of Lemierre syndrome and a septic thrombus the most likely cause of the patient's clinical condition. An infec-

Figure 2. Computed tomography of the neck and soft tissues demonstrating complete occlusion of the right internal jugular (IJ) vein extending from just inferior to the jugular foramen to the lower neck. The black arrows mark the location of the right $\mathrm{IJ}$ vein. The right IJ vein is patent just inferior to the jugular foramen (A), before it begins to narrow at the level of the inferior maxillary sinuses (B) and becomes completely occluded (C) until distally at the level of the lower neck, where it can again be seen filled with contrast (D). A distinct difference can be seen when compared with the left IJ vein.

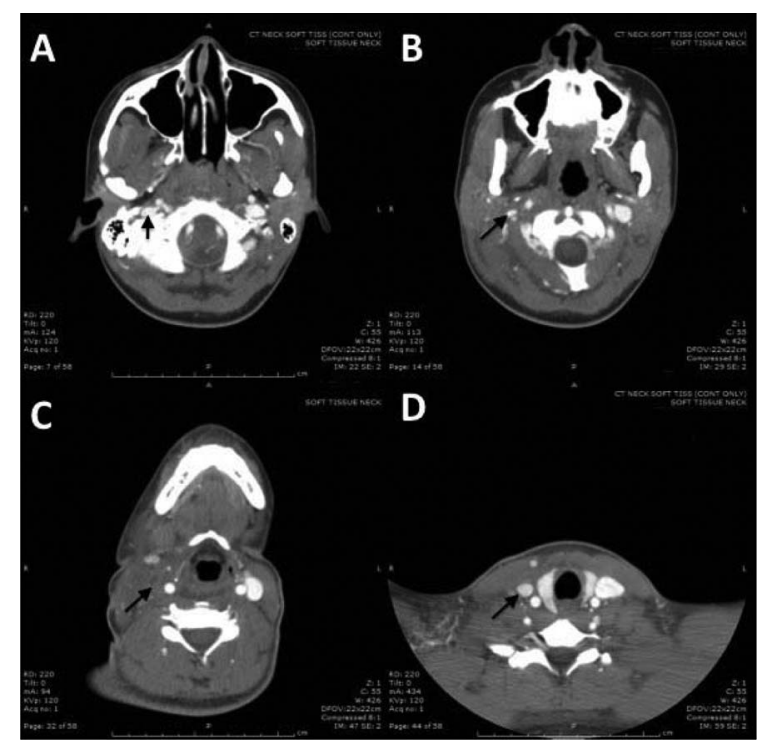

tious disease specialist and an ear, nose, and throat surgeon were consulted. Blood cultures were repeated, and antibiotic coverage was broadened to vancomycin $(1 \mathrm{~g}$ IV loading dose, then $750 \mathrm{mg} \mathrm{IV}$ every 8 hours), metronidazole (500 $\mathrm{mg}$ IV every 8 hours), and high-dose ceftriaxone ( 2 g IV every 24 hours). Because of the risk of septic emboli, increasing symptoms of dyspnea, dry cough, and the incidental finding of bilateral pleural effusions on the initial CT of the neck, a CT angiogram of the chest was ordered to rule out septic pulmonary emboli. The CT angiogram was negative for septic pulmonary emboli but did show moderate-sized bilateral pleural effusions with atelectasis, as shown in Figure 3.

This patient's case is unique in that she never appeared as ill as her condition and the objective findings, including imaging, suggested. By the second hospital day, the patient's sepsis had seemed to resolve with the initial antibiotic treatment and fluid resuscitation. In this clinically stable patient, the risks of attempting to drain the retropharyngeal space seemed to outweigh the benefits, and the patient was placed on a dexamethasone steroid taper $(15,12,8$, and $4 \mathrm{mg}$, once each, every 24 hours; dotted black line in Figure 1), with significant improvement in clinical symptoms.

Vascular surgery also was consulted, and the patient was started on anticoagulation with enoxaparin $(1.5 \mathrm{mg} / \mathrm{kg}$ subcutaneously once daily), bridging to warfarin ( $5 \mathrm{mg}$ daily) on the fifth hospital day. Vancomycin was discontinued on hospital day 6 , and the patient was switched to oral metronidazole. She was discharged from the hospital on hospital day 7 on outpatient IV ceftriaxone ( $2 \mathrm{~g}$ once daily) and metronidazole (500 $\mathrm{mg}$ by mouth every 8 hours). Following discharge, culture sensitivities for $F$. nucleatum came back pan-sensitive to cefotetan, clindamycin, metronidazole, and piperacillin-tazobactam. Follow-up lab values 12 days from admission showed continued improvement (Figure 1). The patient received 2 weeks of IV ceftriaxone before switching to cefpodoxime (400 $\mathrm{mg}$ by mouth twice daily). She continued cefpodoxime and metronidazole for 4 additional weeks. Anticoagulation was continued for a total of 3 months of therapy.

\section{Case Discussion \\ Background}

Sore Throat is a common presenting complaint in the outpatient setting. ${ }^{1}$ Most cases are nonbacterial 
Figure 3. Coronal section of computed tomography angiography of the chest showing moderate-sized bilateral pleural effusions and atelectasis (black arrows).

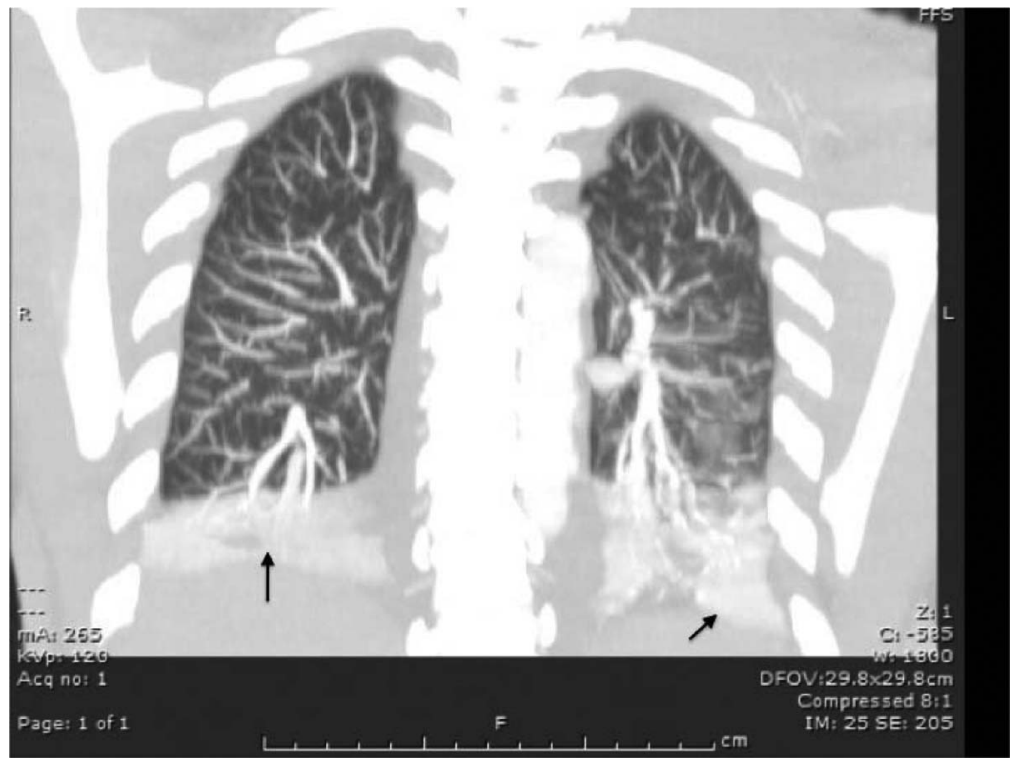

in origin, the others are usually caused by group A eta-hemolytic Streptococcus. ${ }^{2}$ Guidelines exist to help physicians decide whether or not to treat with an antibiotic. ${ }^{3}$ Lemierre syndrome is an exceedingly rare condition characterized by septic thrombophlebitis of the IJ vein. ${ }^{4}$ It also has been referred to as postanginal necrobacillosis and, more recently, as the "forgotten disease." The course of the disease generally starts with a localized oropharyngeal infection, which spreads to involve the lateral pharyngeal space and then the structures of the carotid sheath, eventually causing septic thrombosis of the IJ vein. ${ }^{4,6,7}$ The most commonly identified pathogens are bacteria from the Fusobacterium genus, specifically $F$. necrophorum, which are normal flora within the genitourinary tract, gastrointestinal tract, and oropharynx. ${ }^{4}$ The first report was published by Andre Lemierre in $1936 .^{7}$ While $F$. necrophorum is the most commonly isolated pathogen, multiple other bacteria have been implicated, including other Fusobacterium, Eikenella, Bacterioides, Streptococcus, Peptostreptococcus, Staphylococcus, Porphyromonas, Prevotella, and Proteus spp. Antecedent viral infections also have been associated with this syndrome, including associations with the Epstein-Barr virus. Variants of Lemierre syndrome have been reported, but the discussion here focuses primarily on septic thrombosis of the IJ vein. ${ }^{8}$

The incidence of Lemierre syndrome is extremely rare, with about 0.8 to 1.5 cases per million people annually. ${ }^{5}$ There are some reports of increasing incidence in the recent past, but these have not been shown to be significant; case clusters also have been reported. ${ }^{9}$ It is a disease of the young; most cases occur in individuals ages 14 to 24 years old, with a mean age of 20 years. Other than age and oropharyngeal infection, few risk factors have been clearly identified, although some cases have been associated with a diagnosis of concurrent thrombophilias. ${ }^{10}$

As mentioned previously, Lemierre syndrome generally begins as a localized oropharyngeal infection in the palatine tonsil or peritonsillar tissues. Dental, ear, sinus, and glandular sites are rarer sources of primary infection. ${ }^{4}$ The time interval from primary infection to septic thrombophlebitis is generally 1 to 3 weeks, and mortality occurs in about $5 \% .{ }^{11}$ The exact mechanism is unknown but has been postulated as either hematogenous, local, or via lymphatics.

\section{Diagnosis}

In his initial article, Lemierre ${ }^{7}$ described a syndrome of pyrexial attacks, chills, pulmonary infarcts, and arthritic symptoms several days following an oropharyngeal infection. The diagnosis is often missed until a gram-negative rod is identified in culture. ${ }^{4}$ Patients commonly present with sore throat, neck pain, trismus, fevers, and chills. Upon examination, they classically have increasing asym- 
metric swelling of the neck area overlying the sternocleidomastoid muscle at the angle of the mandible, with associated warmth or induration. However, they can also appear to be septic without any localizing pharyngeal symptoms because the antecedent infection sometimes resolves before presentation. These localized signs and symptoms can be absent or missed during examination.

A high index of suspicion is necessary for a diagnosis of Lemierre syndrome. Clinical signs and symptoms indicating the diagnosis can be few and subtle. In the aforementioned case, the lack of resolution over the expected time frame with ordinary intervention lent itself to the need for a broadened differential diagnosis. Failed treatment, worsening symptoms, or no improvement after 5 days should increase the physician's index of suspicion. ${ }^{12} \mathrm{Be}$ cause Fusobacterium is an anaerobe, blood cultures can take 2 to 7 days $^{13}$ to return positive for infection, further delaying the diagnosis. Prompt diagnosis and initiation of appropriate therapy is crucial in reducing adverse outcomes and significant complications of the disease. There is no mention of the earliest point when imaging is expected to become abnormal, but consideration of the disease and consistent symptoms should prompt confirmation with contrast-enhanced CT of the neck sooner than later. An earlier CT would have sped up the confirmation of our case.

\section{Management}

Like all infections, the optimal antibiotic regimen depends on the source of infection. Initial management should include coverage with broad-spectrum antibiotics. A $\beta$-lactamase-resistant penicillin and an antibiotic with good anaerobic coverage should be included in empiric therapy because of the increasing prevalence of $\beta$-lactamase-producing pathogens. Some Fusobacterium strains are resistant to penicillin. ${ }^{14}$ Acceptable antibiotic regimens include high-dose ceftriaxone along with metronidazole or clindamycin monotherapy. ${ }^{15}$ Vancomycin can be added if suspicion or prevalence of methicillin-resistant Staphylococcus aureus is high.

The optimal duration of therapy is uncertain. Most patients generally require at least 2 to 4 weeks of IV antibiotic therapy followed by another 2 to 4 weeks of oral antibiotics. ${ }^{16}$ Duration of therapy is usually guided by clinical response and the resolution of sepsis. Laboratory studies, including complete blood count, erythrocyte sedimentation rate,
C-reactive protein can be followed to assess response to therapy, but the clinical significance of these and their role in prognosis have not been clearly demonstrated in the literature.

Because of the low incidence of occurrence, no randomized controlled trials of the benefits of anticoagulation exist. Furthermore, evidence is lacking for recommendations on the optimal method of anticoagulation and duration of therapy, though 3 months is the current default duration. ${ }^{17,18}$ Decisions are generally made on a case-by-case basis, with consideration of any contraindications to anticoagulation, risks for bleeding, response to initial antibiotic treatment, risk of complications, and estimated likelihood of thrombus progression or septic emboli. Follow-up CT is done at 1- to 3-month intervals to reevaluate thrombus progression, occurrence of septic emboli, and further need for anticoagulation.

Surgical involvement is usually a last step in the process. Surgery may be indicated for complications of Lemierre syndrome, such as loculated empyemas, brain abscesses, pulmonary abscesses, retropharyngeal abscesses, or other adverse sequelae. Ligation of the IJ vein was a more common practice in the past, but this is rarely performed now. Thrombi that do not extend into the skull generally do not require surgery. Trials of appropriate antibiotic therapy and anticoagulation is the first line of treatment. If no clinical improvement occurs with medical management, then surgery becomes a consideration.

\section{Conclusions and Recommendations}

Lemierre syndrome is a rare disease that requires a high index of clinical suspicion. While it is also known as the "forgotten disease," considering it in a differential diagnosis is crucial because of its high association with serious complications. Prompt diagnosis with early initiation of appropriate treatment is crucial in preventing severe morbidity and mortality. Because of its rarity, specific guidelines and evidence for optimal management do not exist. Antibiotic management should start with broadspectrum coverage that is further guided by immediate blood cultures and CT of the neck and other affected parts, such as the head and chest. Minimal therapy seems to include at least 2 to 4 weeks of IV antibiotics followed by another 2 to 4 weeks of oral therapy. Anticoagulation plays a controversial and 
undefined role in treatment. In light of the serious adverse sequelae of the disease, anticoagulation should be strongly considered in patients with no contraindications or increased risk of bleeding, especially those already demonstrating poor response to appropriate treatment or evidence of septic emboli. The optimal anticoagulation method should be bridge therapy with heparin and the use of warfarin for 3 months. Evaluation of thrombophilias may be indicated, but its clinical benefit is uncertain.

The optimal anticoagulation method, duration of therapy, and duration of follow-up for reimaging is not well defined. Evaluation of thrombophilias may be indicated, but its clinical benefit is uncertain. Because of the lack of evidence to support guidance of management, patients are generally managed on a case-by-case basis, with close monitoring for clinical improvement or adverse sequelae.

The authors give special thanks to the providers involved in this case: Brad Stucky, DO; Brett Hoffecker, MD (Family Medicine, postgraduate year 1 admitting resident); Magaret E. Hagan, MD (Infectious Disease); Glenn Kubina, MD (ENT); Jason Woolard, MD (Vascular Surgery); Jeffrey L. McPherson, MD (Radiology_computed tomography, neck); Thomas D. Cox, MD (Radiology_chest X-ray); Dan Gillespie, MD (Radiology-computed tomography angiography, chest); Dr. Williams, MD, McConnell Air Force Base (Family Medicine, primary care physician).

\section{References}

1. Hing E, Cherry DK, Woodwell DA. National Ambulatory Med Care Survey: 2003 Summary. Adv Data 2005;365:1-48.

2. Bisno AL, Gerber MA, Gwaltney JM Jr, Kaplan EL, Schwartz RH; for the infectious Diseases Society of America. Practice guidelines for the diagnosis and management of group A streptococcal pharyngitis. Clin Infect Dis 2002;35:113-25.

3. Centor RM. Expand the pharyngitis paradigm for adolescents and young adults. Ann Intern Med 2009; 151:812-5.

4. Kuppalli K, Livorsi D, Talati N, Osborn M. Le- mierre's syndrome due to Fusobacterium necrophorum. Lancet Infect Dis 2012;12:808-15.

5. Weesner CL, Cisek JE. Lemierre syndrome: the forgotten disease. Ann Emerg Med 1993;22:256-8.

6. Spelman D. Suppurative (septic) thrombophlebitis. Available from: http://www.uptodate.com/contents/ suppurative-septic-thrombophlebitis? source $=$ search result\&search $=$ Suppurative $+\% 28$ septic $\% 29+$ thrombophlebitis\&selectedTitle $=1 \sim 150$. Accessed March 15, 2013.

7. Lemierre A. On certain septicemias due to anaerobic organisms. Lancet 1936;227:701-3.

8. Akiyama K, Karaki M, Samukawa Y, Mori N. Blindness caused by septic superior ophthalmic vein thrombosis in a Lemierre syndrome variant. Auris Nasus Larynx 2003;40:493-6.

9. Lu MD, Vasavada Z, Tanner C. Lemierre syndrome following oropharyngeal infection: a case series. J Am Board Fam Med 2009;22:79-83.

10. Phan T, So TY. Use of anticoagulation therapy for jugular vein thrombus in pediatric patients with Lemierre's syndrome. Int J Clin Pharm 2012;34: 818-21.

11. Hagelskjaer Kristensen L, Prag J. Lemierre's syndrome and other disseminated Fusobacterium necrophorum infections in Denmark: aprospective epidemiological and clinical survey. Eur J Clin Microbiol Infect Dis 2008;27:779-89.

12. Centor R, Samlowski R. Avoiding sore throat morbidity and mortality: when is it not "just a sore throat"? Am Fam Physician 2011;83:26-8.

13. Riordan T, Wilson M. Lemierre's syndrome: more than historical curiosa. Postgrad Med J 2004;80: 328-34.

14. Baquero F, Reig M. Resistance of anaerobic bacteria to antimicrobial agents in Spain. Eur J Clin Microbiol Infect Dis 1992;11:1016-20.

15. Dool H, Soetekouw R, van Zanten M, Grooters E. Lemierre's syndrome: three cases and a review. Eur Arch Otorhinolaryngol 2005;262:651-4.

16. Armstrong AW, Spooner K, Sanders JW. Lemierre's syndrome. Curr Infect Dis Rep 2000;2:168-73.

17. Hoehn KS. Lemierre's syndrome: the controversy of anticoagulation. Pediatrics 2005;115:1415-6.

18. Hagelskjaer Kristensen L, Prag J. Human necrobacillosis, with emphasis on Lemierre's syndrome. Clin Infect Dis 2000;31:524-32. 